\title{
Hamiltonian memory: An erasable classical bit
}

\author{
Roi Holtzman, Geva Arwas, and Oren Raz $0^{*}$ \\ Department of Physics of Complex Systems, Weizmann Institute of Science, Rehovot, 76100, Israel
}

(Received 28 September 2020; accepted 9 February 2021; published 12 March 2021)

\begin{abstract}
Erasing a bit of information requires probability concentration in phase space, which by Liouville's theorem is impossible in pure Hamiltonian dynamics. It therefore requires dissipative dynamics, leading to the Landauer limit: $k_{B} T \log 2$ of heat dissipation per erasure of one bit. We show that when a conserved quantity confines the dynamic to a single shell with zero thickness, it is possible to concentrate the probability on this shell using Hamiltonian dynamic, and therefore to implement an erasable bit with no thermodynamic cost. This implies that there is no thermodynamic cost associated with bit erasure in the microcanonical ensemble, where the energy of the system is precisely known. However, any uncertainty in the energy results back in the Landauer bound.
\end{abstract}

DOI: 10.1103/PhysRevResearch.3.013232

\section{INTRODUCTION}

In 1961, Landauer established a remarkable relation between information theory and thermodynamics, by arguing that an irreversible computation cannot be made without any energetic cost [1]. Landauer's principle is famously known as the statement that an erasure of one bit of information-the hallmark of irreversible computations-must dissipate at least $k_{B} T \log 2$ of heat, where $k_{B}$ is the Boltzmann constant and $T$ is the temperature of its surrounding environment. This bound is rooted in the dissipative dynamic, enforced by the contraction of the physical system's phase-space volume during the bit erasure. Such an operation cannot be done in an isolated, Hamiltonian dynamic, therefore Landauer concluded that implementing an erasable bit requires a dissipative system. By the second law of thermodynamics, the phase space volume reduction associated with the memory erasure must produce some dissipation, leading to the celebrated Landauer principle.

In the last few decades, Landauer's principle was refined and generalized to various cases. For example, it was generalized for a probabilistic erasure process, i.e., one that only succeeds with some probability [2,3]. Additional generalizations of Landauer's principle include other types of thermodynamic resources such as an angular momentum bath [4], a bound for entropically unbalanced bits [5], unifying the cost of erasing and measuring the bit [6,7], taking into account the mutual information between the bit and the bath [8], $N$ state bit [9], finite time erasure [10,11], and others [12]. All these generalizations, however, rely exclusively on dissi-

\footnotetext{
*oren.raz@weizmann.ac.il

Published by the American Physical Society under the terms of the Creative Commons Attribution 4.0 International license. Further distribution of this work must maintain attribution to the author(s) and the published article's title, journal citation, and DOI.
}

pative dynamics: following Landauer's argument, no energy conserving classical bit was suggested.

The memory technology used today is still far from approaching Landauer's bound. Nevertheless, in recent years several experiments used various physical systems to implement a dissipative bit that can be erased with an energetic cost, which is close to Landauer's bound. These include colloidal particle in a trap [5,13-16], nanomagnetic devices [17-19], superconducting flux bit [20], nuclear spin [21], and single molecule devices [22,23]. Apart from its practical implications, Landauer's principle is commonly used in theoretical studies to resolve seeming violations of the second law [24-28].

In this paper we present an exactly solvable example of a classical Hamiltonian system that can serve as a memory bit, which is erasable at no energetic cost. In this system, the erasure process maps an isolated part of the energy shell onto itself, assuring that the initial and final energies of the system are equal. In addition, the erasure maps most of the points on this isolated part into a small area, enabling us to erase a bit of information without measuring it, and at no thermodynamic cost. We further show that this mapping can be done arbitrarily fast and to an arbitrary accuracy. This implies that contrary to a dissipative bit, such a Hamiltonian bit has no tradeoffs between duration, accuracy, and energetic cost [8]. However, to perform the suggested erasure, the energy of the system has to be known to infinite accuracy: any uncertainty in the energy of the system retrieves the Landauer bound. Therefore, this suggestion is of a purely theoretical nature, as in any realistic situation the knowledge of the system's energy is limited.

\section{HAMILTONIAN BIT: GENERAL DISCUSSION}

To set the ground, let us first review the argument for the inapplicability of a pure Hamiltonian system as an erasable memory device. For a system to serve as a classical memory, there must be a mapping between its physical states and the memory states. For simplicity we assume the memory to be a logical bit, whose memory states (often called logical states) 

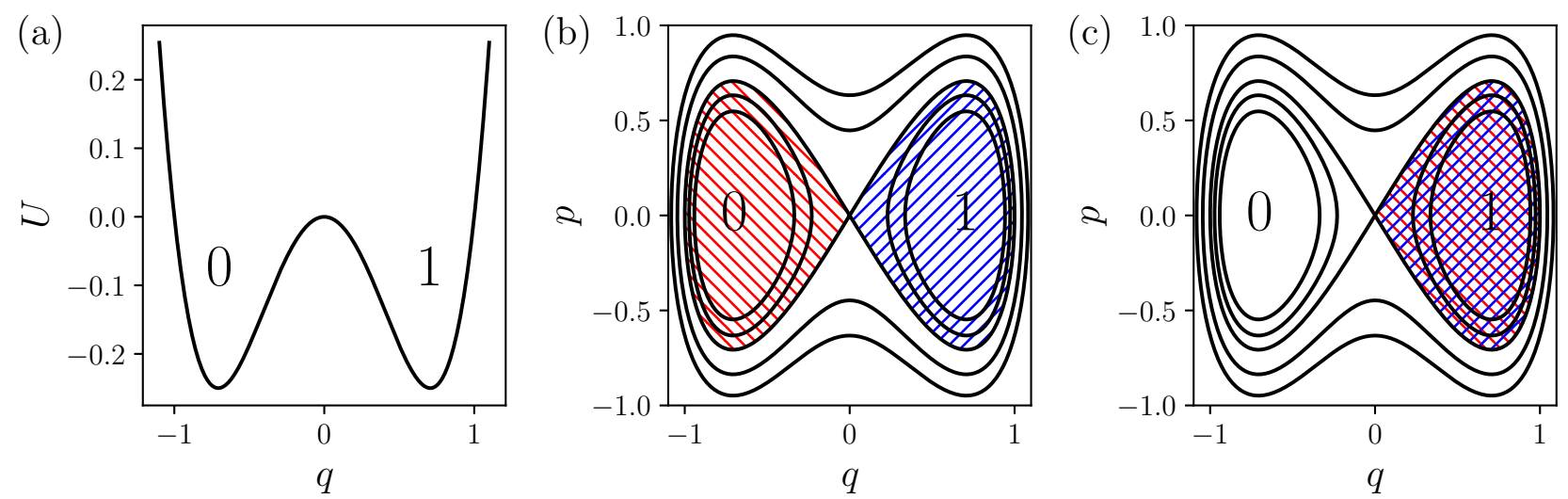

FIG. 1. Restore-to-1 procedure performed on a Hamiltonian double-well system. The right and left wells correspond to the 0 and 1 logical states, respectively. Initially, the bit is unknown - this corresponds to a uniform phase space distribution in the two wells. Restore-to-1 brings the unknown bit to the 1 state. (a) The potential of a double well system. (b) Phase space of the initial configuration which corresponds to the state of an unknown bit: the distribution is evenly split between the 0 (red) and 1 (blue) states. (c) The phase space distribution after a restore-to- 1 process should be concentrated at the state 1 . Both the initial red and blue regions in (b) must end in the red+blue region representing state 1 , therefore the density must increase to compensate volume loss. This is incompatible with Hamiltonian dynamics, due to Liouville's theorem.

are the 0 and 1 states. In addition, to serve as a useful bit the system must have an erasing mechanism. The logic operation of erasure takes any of the logical states, 0 and 1, and sets it to say, 1, at a high enough probability. Such a process is commonly referred as a restore-to- 1 procedure. Note that the procedure has to be performed without measuring the initial state of the bit, as by measuring, the information is copied to an external device, which has to be erased too. Thus an effective erasure mechanism has to be able to erase a bit whose state is unknown. Physically, the restore-to- 1 procedure brings the system to a physical state associated with the logical state 1 [see Figs. 1(a)-1(c)].

Let us consider such a restore-to-1 procedure in Hamiltonian dynamics: As the initial logical state of the bit is unknown, the initial physical state of the bit is unknown as well. The unknown state can be represented by a uniform probability to be in any of the physical states associated with the two logical states 0 and 1 [see Fig. 1(b)]. The erasure operation must concentrate this uniform probability distribution into the physical states associated with the logical state 1 [see Fig. 1(c)]. However, by Liouville's theorem, a Hamiltonian evolution cannot increase phase space probability [29]. Therefore, erasing a bit requires a nonconserving, dissipative dynamic, and is accompanied by a thermodynamic cost.

In what follows, it is shown that a pure Hamiltonian erasable memory device is nevertheless possible, provided that some conserved quantity of the system is known to infinite accuracy. We start with an abstract discussion, followed by a concrete example that demonstrates the idea. Consider a phase space flow that has a stable fixed point, attracting a large volume around it. The evolution under such a flow field can serve as an erasure mechanism, since after long enough time the state of the system is in the vicinity of the fixed point, regardless of where in the attracted volume it was initiated. If the vicinity of the fixed point represents the logical state 1 , then the evolution under this flow generates a restore-to- 1 [see Fig. 2(a)]. Unfortunately, such a flow is incompatible with Hamiltonian dynamics: to satisfy Liouville's theorem and conserve phase space volume, a fixed point which is attractive in one direction must be repelling in some other direction [see Fig. 2(c)]. However, if the evolution of the system is confined to the specific manifold, which is attracted to the fixed point, then the corresponding Hamiltonian can serve as an eraser, even though phase space volume is conserved. Such a confinement can be generated by a conserved quantity; in what follows we use conservation of energy. We denote the manifold of states that are attracted to the fixed point, which is part of the energy shell, by $\mathcal{E}_{\text {ers }}$ [e.g., the violet contour in Fig. 2(c)]. For a time-independent Hamiltonian, a system that is initiated on $\mathcal{E}_{\text {ers }}$ stays on it. Therefore, a Hamiltonian with such a fixed point, denoted by $H_{\text {ers }}$, can serve as an eraser, provided that all of the physical states corresponding to the memory are confined to $\mathcal{E}_{\text {ers }}$.

So far we have described how the erasure is performed. Let us now turn to the physical description of the bit's steady state. When no operation is made on the bit, it evolves under a different, time-independent Hamiltonian, $H_{0}$. The evolution under $H_{0}$ is confined to an energy shell of $H_{0}$ that corresponds to the initial condition. This energy shell might include several disconnected surfaces, and the evolution of the system is confined to the specific surface based on the initial condition. These disconnected surfaces composing the energy shell are referred to as ergodic components, and we denote them by $\mathcal{E}_{0}^{i}$ [see Fig. 2(b) for an example]. To perform erasure, the Hamiltonian is changed from $H_{0}$ to $H_{e r s}$. We have seen that $H_{\text {ers }}$ erases states that are confined to $\mathcal{E}_{\text {ers }}$, thus the evolution under $H_{0}$ must also be confined to $\mathcal{E}_{\text {ers }}$. Therefore, $H_{0}$ must have one of its ergodic components coinciding with $\mathcal{E}_{\text {ers }}$ [compare the violet curves in Figs. 2(b), 2(c)]. For reasons that will become clear later, we denote this ergodic component by $\mathcal{E}_{0}^{+}$. Lastly, the different states in $\mathcal{E}_{0}^{+}$must encode the logical bit in a way that is not altered by $H_{0}$, and such that the vicinity of the fixed point of $H_{\text {ers }}$ encodes the logical state 1.

Let us summarize the construction described above. The system is initially prepared on a specific ergodic component $\mathcal{E}_{0}^{+}$of $H_{0}$. The state of the system in $\mathcal{E}_{0}^{+}$encodes the logical 
(a)

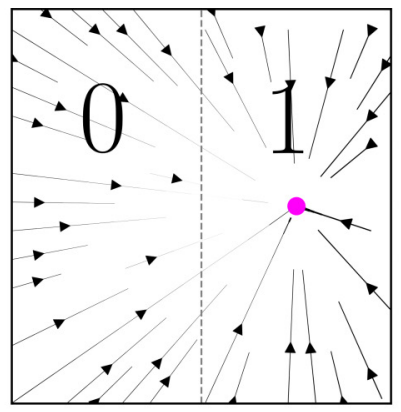

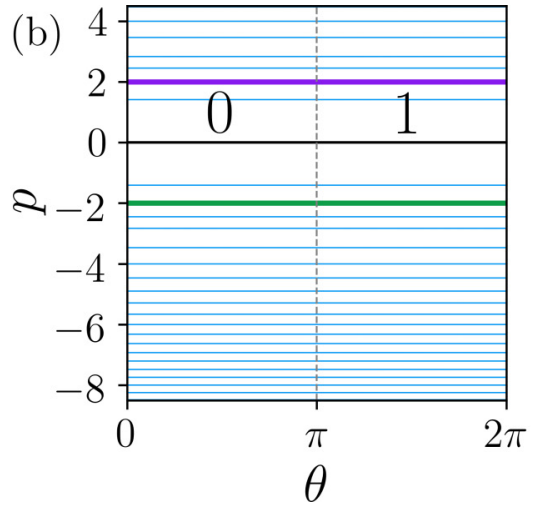

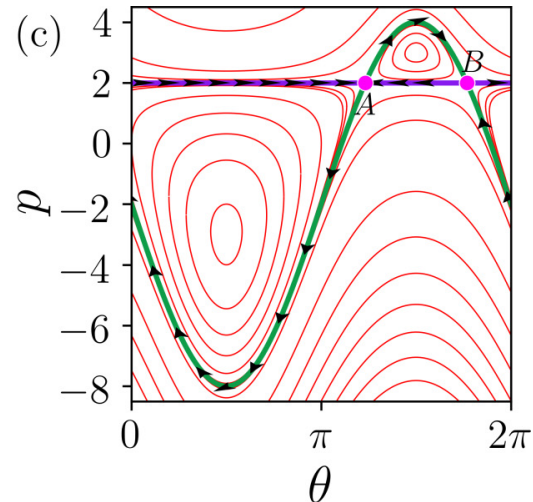

FIG. 2. (a) The flow near an attracting fixed point (magenta) can serve as an eraser: initial conditions representing both the 0 state (left to the dashed line) and the 1 state (right) flow towards the state 1. (b) The phase space structure of the Hamiltonian $H_{0}$ [Eq. (3)]. For $E=2$, the energy shell contains two ergodic components: $\mathcal{E}_{0}^{+}$(violet) and $\mathcal{E}_{0}^{-}$(green). $\mathcal{E}_{0}^{+}$can encode a bit of information; the logical states 1 and 0 are marked. (c) The phase space structure of $H_{\text {ers }}$ in Eqs. (5), (8) with $g=3$ and $E_{0}=2$. The fixed points $A$ and $B$ are on the same energy shell, the green and violet contours. They have both attracting and repelling directions. On $\mathcal{E}_{\text {ers }}$ (the violet contour), all states flow towards the fixed point $A$, except state $B . \mathcal{E}_{0}^{+}$[the violet contour in (b)] is identical to $\mathcal{E}_{\text {ers }}$ [the violet contour in (c)].

bit, and the evolution under $H_{0}$, which is confined to $\mathcal{E}_{0}^{+}$, conserves this logical state. To erase the bit, $H_{\text {ers }}$ is switched on for some time, until large enough portion of $\mathcal{E}_{0}^{+}$is concentrated near the fixed point by the evolution under $H_{\text {ers }}$, and the corresponding logical state is known to a high enough accuracy. At this point, $H_{\text {ers }}$ is switched off, and the system continues to evolve under $H_{0}$.

To generate such a bit, two different Hamiltonians with the relevant overlap between their energy shells are required. Consider first the simplest form of a Hamiltonian,

$$
H=\frac{p^{2}}{2}+U(q)
$$

where we set the mass $m=1$ and $U(q)$ is a positiondependent potential. Two different Hamiltonians $H_{0}$ and $H_{\text {ers }}$ cannot have the desired overlap between their energy shells when both of them are of the form in Eq. (1): the contribution of $p^{2} / 2$ is identical in both Hamiltonians, therefore on $\mathcal{E}_{0}^{+}$the two Hamiltonians must have the same $U(q)$ up to a constant, and both Hamiltonians represent exactly the same physics at this energy and this range of $q$. For the two Hamiltonians to be different and nevertheless have the desired overlap between their energy shells, we consider Hamiltonians that include magnetic fields,

$$
H=\frac{(p-A(q))^{2}}{2}+U(q)
$$

Note that the Hamiltonian in Eq. (1) is symmetric with respect to sign changes of $p$, whereas the one in Eq. (2) is not. An alternative perspective for the same Hamiltonian was presented in Ref. [30], where it was shown that the linear momentum term can be removed to yield a time-dependent Hamiltonian with a standard kinetic energy term and a position-onlydependent potential by a simple transformation, such that the initial and final energy shells are identical.

\section{AN ILLUSTRATIVE EXAMPLE: A PARTICLE ON A RING}

Next, we consider a specific example that illustrates the idea presented above: a particle moving on a ring, with the coordinate $\theta \in[0,2 \pi)$ denoting the angle of the particle on the ring. The Hamiltonian of the particle is given by

$$
H_{0}(\theta, p)=\frac{p^{2}}{2} \text {. }
$$

The phase space of this system is very simple. Each energy shell (except $E=0$ ) is composed of two disjoint ergodic components corresponding to clockwise $(p>0)$ and counterclockwise $(p<0)$ rotations [see Fig. 2(b)]. In what follows we consider the $p>0$ ergodic component of the energy shell, defined as $\mathcal{E}_{0}^{+}=\left\{(\theta, p): H_{0}(p)=E_{0} ; p>0\right\}$. We denote $p_{0}=\sqrt{2 E_{0}}$, so a state with momentum $p_{0}$ belongs to the ergodic component $\mathcal{E}_{0}^{+}$.

To encode one bit of information in the physical state of the particle, which constantly evolves in time, we exploit the periodicity of the particle's motion. The period depends on the exact value of the energy of the system, $E_{0}$. Therefore, for $\mathcal{E}_{0}^{+}$with a period $\tau=2 \pi / p_{0}$, we associate a logical state according to the physical state of the system at stroboscopic time intervals $\tau$, namely at an integer multiplication of $\tau$. A physical state $(\theta, p)$ in a stroboscopic time is assigned a logical state using, for example, the following mapping [see Fig. 2(b)]:

$$
\operatorname{Logic}(\theta, p)=\left\{\begin{array}{ll}
0 & \text { if } \theta \in[0, \pi) \\
1 & \text { if } \theta \in[\pi, 2 \pi)
\end{array} .\right.
$$

The Hamiltonian $H_{0}$ controls the evolution of the bit when no operation is performed on it, and it conserves the encoded logical state. To control the bit, and specifically to perform a restore-to-1 procedure, a different Hamiltonian must be applied on the system for a finite time. Restore-to- 1 means that most initial states $\left(\theta_{i}, p_{i}\right) \in \mathcal{E}_{0}^{+}$are mapped to a final state $\left(\theta_{f}, p_{f}\right) \in \mathcal{E}_{0}^{+}$that encodes the bit 1, i.e., $\operatorname{Logic}\left(\theta_{f}, p_{f}\right)=1$. Note that all final states $\left(\theta_{f}, p_{f}\right)$ must belong to the same 
energy shell $\mathcal{E}_{0}^{+}$, to maintain the logical states of the system. Uncertainty in the energy of the system implies an uncertainty in the stroboscopic time used in the definition of the logical state of the bit, causing the bit to decohere and slowly lose its information.

To erase the bit, we need to find a Hamiltonian, $H_{\text {ers }}(\theta, p, t)$, that has three properties: (i) It maps the specific ergodic component $\mathcal{E}_{0}^{+}$into itself in a finite time; (ii) It concentrates the uniform distribution on this energy shell; and (iii) It is physically legitimate. In what follows, we refer to a Hamiltonian as having property (iii) if it has the form given in Eq. (2) associated with a magnetic field. Although there are many Hamiltonians that satisfy all these constraints, finding one of them is not a trivial task. Next, we show a systematic method to find such a Hamiltonian, by interpolating between $H_{0}$ and a different Hamiltonian that shares the ergodic component $\mathcal{E}_{0}^{+}$with $H_{0}$.

Let us formulate the conditions (i) and (ii) in terms of $\theta$ and $p$. Condition (i) is satisfied if any initial state $\left(\theta_{i}, p_{i}=\right.$ $\left.\sqrt{2 E_{0}}\right) \in \mathcal{E}_{0}^{+}$evolves under $H_{\text {ers }}$ to a point $\left(\theta_{f}, p_{f}\right)$ with the same momentum value, namely $p_{f}=\sqrt{2 E_{0}}$. A necessary condition for (ii) is that the coordinate velocity $\dot{\theta}$ is a function of $\theta$. Otherwise, all the coordinates evolve with the same rate, and the uniform distribution remains a uniform distribution.

To generate probability concentration we add a term to the Hamiltonian, denoted by $H_{\text {con }}$. The full erasure Hamiltonian is given by

$$
H_{\text {ers }}(\theta, p, t)=H_{0}(p)+g(t) H_{\mathrm{con}}(\theta, p),
$$

where $g(t)$ is a function that ramps the concentration term $H_{\text {con }}$ on and off in a continuous manner and controls the concentration magnitude. With this Hamiltonian, the equations of motion are

$$
\begin{gathered}
\dot{p}=-\frac{\partial H_{\text {ers }}}{\partial \theta}=-g(t) \frac{\partial H_{\mathrm{con}}}{\partial \theta} \\
\dot{\theta}=\frac{\partial H_{\text {ers }}}{\partial p}=p+g(t) \frac{\partial H_{\mathrm{con}}}{\partial p} .
\end{gathered}
$$

One way to meet condition (i) is to have $\left.\frac{\partial H_{\text {con }}}{\partial \theta}\right|_{p_{0}}=0$, which implies that $p_{0}$ is constant. In this case, $\mathcal{E}_{0}^{+}$is part of the energy shell of $H_{\text {ers }}$ too.

A choice for $H_{\text {con }}$ that satisfies conditions (i), (ii), and (iii) is

$$
H_{\text {con }}(\theta, p)=\left(p-\sqrt{2 E_{0}}\right) \sin (\theta) .
$$

On $\mathcal{E}_{0}^{+}$we have $p=\sqrt{2 E_{0}}$, so $H_{\text {con }}=0$. Therefore it is $\theta$ independent on $\mathcal{E}_{0}^{+}$, and thus $p$ is conserved on this ergodic component [see Eq. (6)]. In addition, $H_{\text {con }}$ has only a linear term in $p$ which can be brought to the form of Eq. (2), and therefore it fulfills condition (iii).

Let us next show that $H_{\text {con }}$ provides the desired probability concentration, as required by condition (ii). The equations of motion for the full erasure Hamiltonian on the specific ergodic component $\mathcal{E}_{0}^{+}$for the specific choice of $H_{\text {ers }}$ are given by

$$
\begin{gathered}
\left.\dot{p}\right|_{p_{0}}=0 \\
\left.\dot{\theta}\right|_{p_{0}}=p_{0}+g(t) \sin \theta .
\end{gathered}
$$
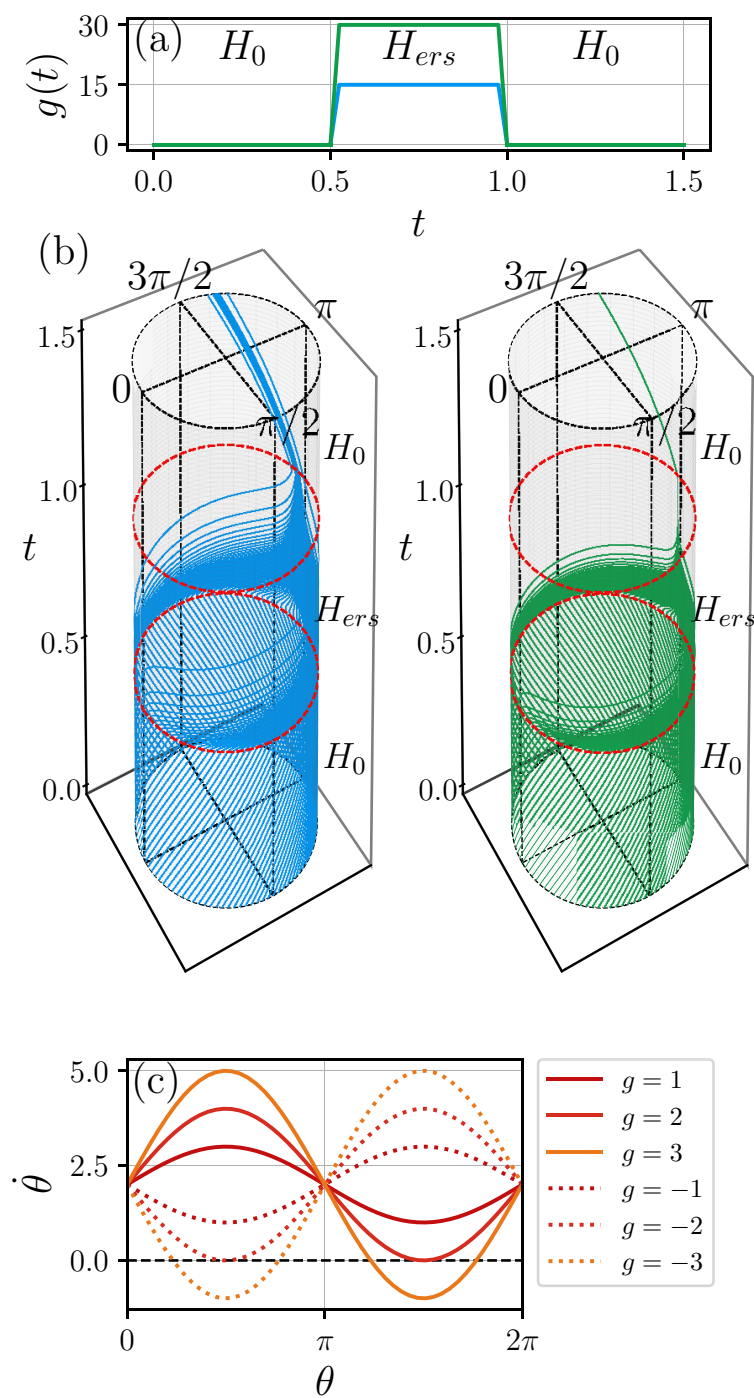

FIG. 3. (a) The protocols $g(t)$ used in $H_{e r s}(t)$, for the evolution in (b). (b) Evolution under $H_{\text {ers }}(t)$ of 100 uniformly distributed initial angles. The angle $\theta$ is represented as the angle on the unit circle, and the time evolution is along the vertical axis. For $0 \leqslant t<0.5$ the system evolves under $H_{0}$, and therefore the distribution remains uniform. From $t=0.5$ to $t=1$, a restore-to- 1 protocol is applied, with $g(t)$ shown in (a). The left panel corresponds to maximal value $\max g(t)=15$ (blue), and the right panel to $\max g(t)=30$ (green). At the end of these protocols, at $t=1$, all the angles are extremely concentrated around one value $\theta_{s}$. Then the bit continues to evolve under $H_{0}$ for $1 \leqslant t<1.5$, during which the concentrated distribution rotates at a constant velocity. Evidently, larger $g$ results in a faster and more accurate erasure. (c) $\dot{\theta}$ as a function of $\theta$ given in Eq. (10), for a few values of $g$. For $g>\sqrt{2 E_{0}}=2$, a stable and an unstable fixed points exist and the microcanonical distribution is singular.

The evolution of any angle $\theta_{i} \in \mathcal{E}_{0}^{+}$is clear from Eq. (10). For $g(t)>p_{0}, \dot{\theta}$ vanishes twice at $\theta_{s}$ and $\theta_{u}$ which correspond to stable and unstable fixed points of the dynamic of the angles [see Fig. 3(c) for its plot at several values of $g$ ]. Therefore, for $g(t)>p_{0}$ the dynamic has a fixed point on $\mathcal{E}_{0}^{+}$, and all angles flow towards the stable angle $\theta_{s}$. Moreover, for large enough $g(t), \dot{\theta}$ scales with $g(t)$ for most values of $\theta$. Thus, most initial 
conditions flow very rapidly to $\theta_{s}$. This ensures that almost all initial angles converge to $\theta_{s}$ provided that the duration protocol $\mathcal{T}_{\text {ers }}$ and the magnitude of $g(t)$ are both large enough. Moreover, it shows that there is no fundamental bound on the rate of probability concentration, and that the concentration, namely the probability to find the system in a state that corresponds to the desired bit, can be arbitrarily high. Indeed, for any arbitrarily small erasure time $\mathcal{T}_{\text {ers }}$, one can choose large enough $g(t)$, such that almost all initial angles $\theta_{i} \in \mathcal{E}_{0}^{+}$are mapped arbitrarily close to the stable angle $\theta_{s} \in \mathcal{E}_{0}^{+}$. However, not all initial angles can be mapped to $\theta_{s}$, since the ring topology of $\mathcal{E}_{0}^{+}$is invariant by the dynamic, and thus cannot be torn. Nevertheless, the fraction of initial angles mapped close to $\theta_{s}$ can be increased arbitrarily by increasing $g(t)$. Lastly, we note that large enough $g(t)>0$ results in $\theta_{s}>\pi$, whereas small enough $g(t)<0$ results in $\theta_{s}<\pi$, therefore by controlling the sign of $g(t)$ both restore-to- 1 and restore-to- 0 can be implemented.

To show the convergence of most initial conditions, 100 uniformly spaced initial angles $\left\{\theta_{j}\right\}_{j=1}^{100} \in[0,2 \pi)$ were chosen in Fig. 3, and evolved according to a specific protocol: for $0 \leqslant t<0.5$ the system evolved under $H_{0}$ with $E_{0}=2$, so the angles evolved uniformly. During $0.5 \leqslant t<1$ the erasure is performed by $H_{e r s}(t)$ as defined above. The specific choice of $g(t)$ is shown in the top panel of the figure. Lastly, the bit evolved again under $H_{0}$ for $1 \leqslant t<1.5$. The left and right central panels show the evolution of $\theta_{j}(t)$, with different amplitudes for $g(t)$. In agreement with the analysis above all of the trajectories end up with almost the same final (stable) angle $\theta_{s}$. Moreover, the rate of convergence increases with the magnitude of $g(t)$.

To quantify the fidelity of the erasure, we examine the Shannon entropy of the logical states 0 and 1 given by

$$
\mathcal{S}\left(\left\{p_{0}, p_{1}\right\}\right)=-\sum_{i=0}^{1} p_{i} \log p_{i},
$$

where $p_{i}$ is the probability of the bit to have the value $i \in$ $\{0,1\}$. Initially the bit is uniformly distributed and therefore $\mathcal{S}_{i}=\log 2$. For the specific protocols implemented in Fig. 3, the final entropy values are $\mathcal{S}_{f} \approx 0.023$ for $\max g(t)=15$, and $\mathcal{S}_{f} \approx 6.2 \times 10^{-5}$ for $\max g(t)=30$. This decrease in the information entropy comes with no energetic cost, as the initial and final states of the system are on the same energy shell, so no work is done on the system. Moreover, the protocol can be repeatedly applied on as many bits as needed without any measurement or any other type of thermodynamic cost.

Does the above construction violate the second law? To see why this is not the case, we note that the Hamiltonian dynamic is reversible. In other words, even after the erasure process, it is in principle possible to reverse the dynamic and find the initial state of the system. Therefore, no physical entropy is generated in the erasure process. However, the logical state is irreversible: if only the logical state after the erasure is known, namely that the system represents the logical state 1 , then there is no way to know what was the previous state of the system. Logical irreversible process with a reversible dynamic was already pointed out by Sagawa in Ref. [8], where he argued that the combination of the bit and a thermal bath together is a closed system with a reversible dynamic.

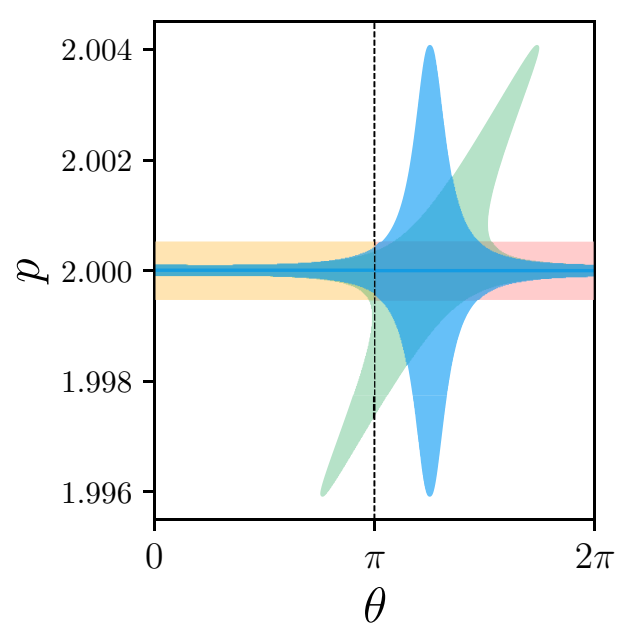

FIG. 4. A restore-to-1 procedure performed on a thick energy shell. The initial shell is illustrated by the colors yellow and red which indicate the logical states 0 and 1 respectively. The restoreto- 1 results in the blue volume which is concentrated around some value of $\theta$ and has a large spread in $p$. The parameters taken are, $E_{0}=2, \delta E=0.001, \max g(t)=3$, where the total duration of the protocol is 1 and the fraction of time taking for the ramp up and ramp down of the protocol $g(t)$ is 0.25 [see Fig. 3(a)]. The crucial point is that the blue volume is not confined to the initial thick shell. Therefore, the erasure is not cyclic. The green volume is the time evolution by $H_{0}$ of the blue volume. Namely, this is the evolution of the bit when it is idle. The shearing effect coming from the different values of $p$ is apparent and demonstrates how the information is lost after some time. In this plot the idle time is $30 \tau$.

\section{FINITE WIDTH SHELL}

The erasure procedure discussed above works as long as the system is initiated with a specific energy value. Indeed, on a specific energy shell it is possible to induce, through a time-dependent Hamiltonian, an effective compressible flow and thus to change the phase space distribution on the shell. In order to induce this compression, the erasure Hamiltonian $H_{\text {ers }}$ has to be tailored precisely to $\mathcal{E}_{0}^{+}$[note the dependence of $H_{\text {con }}$ on $E_{0}$ in Eq. (8)]. This means that the energy of the system $E_{0}$ has to be known precisely. In reality, however, there is always some finite accuracy at which the energy can be known: at the very least, the quantum uncertainty principle sets a minimal bound on the accuracy at which the energy is known, based on the duration of its measurement. Such a finite accuracy corresponds to a thick energy shell that has some nonzero volume in phase space.

In the case of a thick energy shell, squeezing the distribution in the $\theta$ direction generates expansion in the $p$ direction due to conservation of phase space volume, as illustrated in Fig. 4. There are several consequences to this expansion: (i) By Eq. (7) a spread in the $p$ direction translates with time into a spread in the $\theta$ direction, even for $g(t)=0$, namely this shearing exists even for $H_{0}$, if different points have different $p$ values. The spreading in the degree of freedom that encodes the information, $\theta$ in our case, eventually causes the bit to lose the encoded information, see Fig. 4; (ii) The energetic cost of erasing the bit generically does not vanish in this case, as the initial and final energies are not identical; and (iii) The set 
of final states is not contained by the set of initial states, as demonstrated in Fig. 4. Therefore, the above analysis of a single erasure procedure does not capture the average energetic cost of many cycles, and additional analysis is necessary.

Let us discuss first the spread in $\theta$ : the dynamic of $\theta$ under $H_{0}$ is given by

$$
\dot{\theta}=p .
$$

When the system is confined to $\mathcal{E}_{0}^{+}$, all the states have the same value for $p$, and hence $\dot{\theta}$ is constant under the evolution of $H_{0}$ and the information does not decohere with time. However, a spread in $p$ shears the distribution and spreads it in the $\theta$ direction. This shear is demonstrated in Fig. 4, where the green area is the time evolution under $H_{0}$ of the blue area after $30 \tau$. This shear limits the lifetime of the bit since a distribution that is localized around some value of $\theta$ would spread after long enough time. Finite lifetime is not a property of Hamiltonian bits only: in dissipative bits, thermal fluctuations can change the state of the bit, so they too have a finite lifetime. Therefore, by itself this is not a limit on costless Hamiltonian bits.

Next, let us consider the energetic cost of the proposed erasure for a thick energy shell: in this case the initial condition is sampled uniformly from the set $\delta \mathcal{E}_{0}^{+}=\left\{(\theta, p): H_{0}(p) \in\left(E_{0}+\delta E / 2, E_{0}-\delta E / 2\right), p>0\right\}$. The equations of motion (6), (7) define a transformation $M$ that maps an initial state $\left(\theta_{i}, p_{i}\right)$ to a final state $\left(\theta_{f}, p_{f}\right)$. For small $\delta E$, the spread in momentum $\delta p=\delta E / p_{0}$ is small too, and thus the transformation $M\left(\theta_{i}, p_{i}\right)$ can be expanded around $\left(\theta_{i}, p_{0}\right)$

$$
\begin{aligned}
M\left(\theta_{i}, p_{0}+\delta p\right) & \approx M\left(\theta_{i}, p_{0}\right)+\delta p \frac{\partial M}{\partial p}\left(\theta_{i}, p_{0}\right) \\
& =\left(\theta_{f}, p_{0}\right)+m\left(\theta_{i}, p_{0}\right) \delta p .
\end{aligned}
$$

Therefore the initial energy shell $\delta \mathcal{E}_{0}^{+}$is transformed by $M$ to a distorted volume given by $\delta \mathcal{E}_{f}=$ $\left\{(\theta, p): p \in\left(p_{0}-m(\theta) \delta p / 2, p_{0}+m(\theta) \delta p / 2\right)\right\}$. The average work performed during the restore-to- 1 procedure is given by

$$
\begin{aligned}
\langle W\rangle & =\left\langle E_{f}\right\rangle-\left\langle E_{i}\right\rangle \\
& =\iint_{\delta \mathcal{E}_{f}} P(\theta, p) E(p) d \theta d p-\iint_{\delta \mathcal{E}_{0}^{+}} P(\theta, p) E(p) d \theta d p .
\end{aligned}
$$

In both integrals the probability distribution $P(\theta, p)$ is uniform: Initially, the distribution is uniform, representing equal probabilities for all the corresponding states. The Hamiltonian evolution $M$ conserves the probability distribution. Therefore only the limits of integration change. Expanding Eq. (14) in orders of $\delta p$ one finds that the zeroth order vanishes since the volumes of $\delta \mathcal{E}_{0}^{+}$and $\delta \mathcal{E}_{f}$ are equal. The linear term vanishes for each integral separately due to the symmetry of $p$ around $p_{0}$. Therefore, the first nonvanishing term is quadratic in $\delta p$, and so quadratic in $\delta E$. Note that by reducing $\delta E$ one can reduce the work performed in this restore-to- 1 procedure arbitrarily close to zero. We thus conclude that although there is some nonzero cost associated with the erasure protocol for a thick energy shell, this is not the reason why such a system is bounded by the Landauer principle. We also note that the above analysis holds for small enough $\delta E$. However, if $\delta E$ grows after each erasure procedure, then at some point this analysis becomes invalid.

To understand why a bit implemented in a thick energy shell is nevertheless subject to the Landauer bound, we note that the concentration of probability in the $\theta$ direction implies that the set of final states is not confined in the set of initial states, as shown in Fig. 4. Let us consider two alternatives for the cyclic operation of the bit: (i) Bringing the bit back to be confined in the set of initial states $\delta \mathcal{E}_{0}^{+}$; (ii) Keep using the bit again and again without confining it to $\delta \mathcal{E}_{0}^{+}$. To bring back all states of the bit to $\delta \mathcal{E}_{0}^{+}$such that it encodes the logical state 1 (i.e., the red volume in Fig. 4) we must either throw away the part of the blue volume, which is out of bounds, or compress the phase space volume. Removing some part of the phase space volume is obviously not sustainable. We must therefore compress phase space volume, which amount to Landauer's bound. Let us consider option (ii). In this case the restore-to- 1 operation is applied on the same bit, which now has a larger spread in the $p$ direction. Therefore, after every iteration the energy thickness of the set of possible states grows. As the energy is bounded from below but not from above, it is impossible to increase the thickness of the energy shell without changing the average energy, and therefore at some point erasing the bit cost energy, which quite quickly becomes even larger than the Landauer bound.

\section{v. CONCLUSIONS}

To conclude, we have shown with a simple solvable example, that a perfectly isolated energy conserving system can serve as a mutable memory. However, in order to implement a restore-to- 1 procedure properly by Hamiltonian dynamics, the ergodic component on which the system evolves must be known precisely. In our example this corresponds to knowing the energy of the system to infinite accuracy. In realistic situations, there is always some finite accuracy in which properties as the energy can be measured, therefore this approach cannot be implemented in reality. Nevertheless, it reveals some theoretical aspects regarding the microcanonical ensemble. Namely that the uniform distribution, which corresponds to the distribution with maximal entropy for the microcanonical ensemble, can be altered at no energetic cost, and remain indefinitely in this altered distribution. This is in line with other peculiarities of the microcanonical ensemble, such as extracting energy on average by performing a cyclic process [31] and building microcanonical Szilard engines [25,28].

The construction used in this paper keeps the state of the system on a specific energy shell at all time, and uses a fixed point in $H_{e r s}$ to concentrate the probability distribution. We note that these are not essential to construct an erasure: concentration of probability occurs even if $H_{\text {ers }}$ does not have a fixed point as long as $\mathcal{E}_{0}^{+}$coincides with $\mathcal{E}_{\text {ers }}$ and the microcanonical distribution on $\mathcal{E}_{0}^{+}$is different for $H_{0}$ and $H_{\text {ers }}$. Alternatively, there is no need for the dynamic to stay confined on $\mathcal{E}_{0}^{+}$at all times when $H_{\text {ers }}$ operates: it is enough that $H_{\text {ers }}$ maps $\mathcal{E}_{0}^{+}$onto itself at a specific time. Lastly, we note that other conserved quantities can be used for a similar construction. 


\section{ACKNOWLEDGMENTS}

We would like to thank Asaf Miron, Grisha Falkovich, Hillel Aharoni, Ugur Cetiner, John Bechhoefer, and Chris Jarzynski for useful discussions. O.R. receives the support of the Shlomo and Michla Tomarin career development chair, and is supported by the Abramson Family Center for Young Scientists and by the Israel Science Foundation, Grant No. 950/19.
[1] R. Landauer, Irreversibility and heat generation in the computing process, IBM J. Res. Dev. 5, 183 (1961).

[2] O. J. E. Maroney, Generalizing Landauer's principle, Phys. Rev. E 79, 031105 (2009).

[3] L. Gammaitoni, Beating the Landauer's limit by trading energy with uncertainty, arXiv:1111.2937.

[4] J. A. Vaccaro and S. M. Barnett, Information erasure without an energy cost, Proc. R. Soc. A 467, 1770 (2011).

[5] M. Gavrilov and J. Bechhoefer, Erasure without Work in an Asymmetric Double-Well Potential, Phys. Rev. Lett. 117, 200601 (2016).

[6] T. Sagawa and M. Ueda, Minimal Energy Cost for Thermodynamic Information Processing: Measurement, and Information Erasure, Phys. Rev. Lett. 102, 250602 (2009).

[7] A. B. Boyd and J. P. Crutchfield, Maxwell Demon Dynamics: Deterministic Chaos, the Szilard Map, and the Intelligence of Thermodynamic Systems, Phys. Rev. Lett. 116, 190601 (2016).

[8] T. Sagawa, Thermodynamic, and logical reversibilities revisited, J. Stat. Mech.: Theory Exp. (2014) P03025.

[9] E. Bormashenko, Generalization of the Landauer principle for computing devices based on many-valued logic, Entropy 21, 1150 (2019).

[10] K. Proesmans, J. Ehrich, and J. Bechhoefer, Finite-Time Landauer Principle, Phys. Rev. Lett. 125, 100602 (2020).

[11] K. Proesmans, J. Ehrich, and J. Bechhoefer, Optimal finitetime bit erasure under full control, Phys. Rev. E 102, 032105 (2020).

[12] D. H. Wolpert, The stochastic thermodynamics of computation, J. Phys. A: Math. Theor. 52, 193001 (2019).

[13] A. Bérut, A. Arakelyan, A. Petrosyan, S. Ciliberto, R. Dillenschneider, and E. Lutz, Experimental verification of Landauer's principle linking information, and thermodynamics, Nature (London) 483, 187 (2012).

[14] Y. Jun, M. Gavrilov, and J. Bechhoefer, High-Precision Test of Landauer's Principle in a Feedback Trap, Phys. Rev. Lett. 113, 190601 (2014).

[15] A. Bérut, A. Petrosyan, and S. Ciliberto, Information, and thermodynamics: experimental verification of Landauer's erasure principle, J. Stat. Mech.: Theory Exp. (2015) P06015.

[16] M. Gavrilov, R. Chétrite, and J. Bechhoefer, Direct measurement of weakly nonequilibrium system entropy is consistent with Gibbs-Shannon form, Proc. Natl. Acad. Sci. 114, 11097 (2017).

[17] J. Hong, B. Lambson, S. Dhuey, and J. Bokor, Experimental test of Landauer's principle in single-bit operations on nanomagnetic memory bits, Sci. Adv. 2, e1501492 (2016)
[18] L. Martini, M. Pancaldi, M. Madami, P. Vavassori, G. Gubbiotti, S. Tacchi, F. Hartmann, M. Emmerling, S. Höfling, L. Worschech et al., Experimental, and theoretical analysis of Landauer erasure in nano-magnetic switches of different sizes, Nano Energy 19, 108 (2016).

[19] R. Gaudenzi, E. Burzurí, S. Maegawa, H. S. J. van der Zant, and F. Luis, Quantum landauer erasure with a molecular nanomagnet, Nat. Phys. 14, 565 (2018).

[20] O.-P. Saira, M. H. Matheny, R. Katti, W. Fon, G. Wimsatt, J. P. Crutchfield, S. Han, and M. L. Roukes, Nonequilibrium thermodynamics of erasure with superconducting flux logic, Phys. Rev. Research 2, 013249 (2020).

[21] J. P. S. Peterson, R. S. Sarthour, A. M. Souza, I. S. Oliveira, J. Goold, K. Modi, D. O. Soares-Pinto, and L. C. Céleri, Experimental demonstration of information to energy conversion in a quantum system at the Landauer limit, Proc. R. Soc. A 472 , 20150813 (2016).

[22] L. L. Yan, T. P. Xiong, K. Rehan, F. Zhou, D. F. Liang, L. Chen, J. Q. Zhang, W. L. Yang, Z. H. Ma, and M. Feng, Single-Atom Demonstration of the Quantum Landauer Principle, Phys. Rev. Lett. 120, 210601 (2018).

[23] U. Cetiner, O. Raz, and S. Sukharev, Dissipation during the gating cycle of the bacterial mechanosensitive ion channel approaches the Landauer's limit, doi: 10.1101/2020.06.26.174649.

[24] C. H. Bennett, The thermodynamics of computation-a review, Int. J. Theor. Phys. 21, 905 (1982).

[25] S. Vaikuntanathan and C. Jarzynski, Modeling Maxwell's demon with a microcanonical Szilard engine, Phys. Rev. E 83, 061120 (2011).

[26] J. Bergli, Accuracy of energy measurement, and reversible operation of a microcanonical Szilard engine, Phys. Rev. E 89, 042120 (2014).

[27] J. M. R. Parrondo, J. M. Horowitz, and T. Sagawa, Thermodynamics of information, Nat. Phys. 11, 131 (2015).

[28] R. Marathe and J. M. R. Parrondo, Cooling Classical Particles with a Microcanonical Szilard Engine, Phys. Rev. Lett. 104, 245704 (2010).

[29] H. Goldstein, C. Poole, and J. Safko, in Classical Mechanics (Addison-Wesley, 2002), pp. 782-783.

[30] C. Jarzynski, S. Deffner, A. Patra, and Y. Subaşı, Fast forward to the classical adiabatic invariant, Phys. Rev. E 95, 032122 (2017).

[31] K. Sato, An example of a mechanical system whose ensemble average energy, starting with a microcanonical ensemble, decreases after an operation, J. Phys. Soc. Jpn. 71, 1065 (2002). 